\title{
Cleavage of ST6Gal I by Radiation-Induced BACE1 Inhibits Golgi-Anchored ST6Gal I-Mediated Sialylation of Integrin $\beta 1$ and Migration in Colon Cancer Cells
}

Minyoung Lee ${ }^{1+}$, Jung-Jin Park ${ }^{1,2+}$, Young-Gyu Ko ${ }^{2^{*+}}$ and Yun-Sil Lee ${ }^{3^{*+}}$

\begin{abstract}
Background: Previously, we found that $\beta$-galactoside $\alpha 2,6$-sialyltransferase (ST6Gal I), an enzyme that adds sialic acids to N-linked oligosaccharides of glycoproteins and is frequently overexpressed in cancer cells, is up-regulated by ionizing radiation (IR) and cleaved to a form possessing catalytic activity comparable to that of the Golgilocalized enzyme. Moreover, this soluble form is secreted into the culture media. Induction of ST6Gal I significantly increased the migration of colon cancer cells via sialylation of integrin $\beta 1$. Here, we further investigated the mechanisms underlying ST6Gal I cleavage, solubilization and release from cells, and addressed its functions, focusing primarily on cancer cell migration.

Methods: We performed immunoblotting and lectin affinity assay to analyze the expression of ST6 Gal I and level of sialylated integrin $\beta 1$. After ionizing radiation, migration of cells was measured by in vitro migration assay. $\alpha 2,6$ sialylation level of cell surface was analyzed by flow cytometry. Cell culture media were concentrated and then analyzed for soluble ST6Gal I levels using an $\alpha 2,6$ sialyltransferase sandwich ELISA.

Result: We found that ST6Gal I was cleaved by BACE1 ( $\beta$-site amyloid precursor protein-cleaving enzyme), which was specifically overexpressed in response to IR. The soluble form of ST6Gal I, which also has sialyltransferase enzymatic activity, was cleaved from the Golgi membrane and then released into the culture media. Both noncleaved and cleaved forms of ST6Gal I significantly increased colon cancer cell migration in a sialylation-dependent manner. The pro-migratory effect of the non-cleaved form of ST6Gal I was dependent on integrin $\beta 1$ sialylation, whereas that of the cleaved form of ST6Gal I was not, suggesting that other intracellular sialylated molecules apart from cell surface molecules such as integrin $\beta 1$ might be involved in mediating the pro-migratory effects of the soluble form of ST6Gal I. Moreover, production of soluble form ST6Gal I by BACE 1 inhibited integrin $\beta 1$ sialylation and migration by Golgi-anchored form of ST6Gal I.
\end{abstract}

Conclusions: Our results suggest that soluble ST6Gal I, possibly in cooperation with the Golgi-bound form, may participate in cancer progression and metastasis prior to being secreted from cancer cells.

Keywords: BACE1, Migration, Radiation, ST6Gal I

\footnotetext{
*Correspondence: ygko@korea.ac.kr; yslee0425@ewha.ac.kr

+ Contributed equally

${ }^{2}$ College of Life Sciences and Biotechnology, Korea University, 1, 5-ka,

Anamdong, Sungbuk-gu, Seoul 136-701, South Korea

${ }^{3}$ College of Pharmacy \& Division of Life \& Pharmaceutical Sciences, Ewha

Womans University, 11-1 Daehyun-Dong, Seodaemun-Gu, Seoul 120-750,

South Korea

Full list of author information is available at the end of the article
} 


\section{Background}

ST6Gal I ( $\beta$ galactoside $\alpha 2,6$ sialyltransferase, CMPNeuAc: Gal $\beta(1,4)$ GlcNAc: $\alpha 2,6$ sialyltransferase) is an important glycosyltransferase that adds a sialic acid residue to the terminal position on $\mathrm{N}$-linked oligosaccharides [1,2]. It is localized in the Golgi apparatus in a membrane-anchored form and is cleaved into a secretary protein by cathepsin-like proteases [3]. Recent studies and clinical reports have emphasized the importance of ST6Gal I in cancer progression and metastasis. ST6Gal I is up-regulated in colon adenocarcinoma and its expression is positively associated with tumor cell migration and invasion [4-6]. Specifically, patients with metastasizing tumors have high levels of ST6Gal I in their serum, and serum levels of ST6Gal I are correlated with the progression of colorectal carcinomas and cancer metastasis [7-13]. However, a possible biological role of ST6Gal I in the plasma has not been reported.

Metastasis represents an obligatory step in cancer progression. A variety of molecules contribute to cancer progression and metastasis [14], and many of the factors that function in tumor metastasis are glycoproteins [15-17]. It has been previously demonstrated that integrin $\beta 1$ is a major substrate of ST6Gal I $[4,18]$. In colon epithelial cells, oncogenic Ras has been shown to up-regulate ST6Gal I expression, leading to increased $\alpha-2,6$ sialylation of $\beta 1$ integrin [19]. Hypersialylation of integrin $\beta 1$ augments colon cancer metastasis by altering cellular preference for a certain extracellular matrix milieu as well as by stimulating cell migration. Integrins also regulate cellular functions, including survival, proliferation and cell spreading, through the function of signaling molecules co-localized to the focal adhesion complex $[20,21]$. We have previously demonstrated that exposure to ionizing radiation (IR) increases the expression of ST6Gal I as well as the level of sialylated glycoprotein. Sialylation of integrin $\beta 1$ by exposure of cells to IR increases the adhesion and migration of colon cancer cells through integrin $\beta 1$-mediated cellular signaling. Therefore, integrin $\beta 1$ sialylation and the subsequent activation of p130CAS, paxillin, and AKT signaling may be one of the mechanisms involved in IR-mediated-radioresistance and cancer metastasis [22-26].

$\beta$-site amyloid precursor protein-cleaving enzyme (BACE) is a membrane-bound aspartic protease that cleaves the amyloid precursor protein (APP) in the pathogenesis of Alzheimer's disease [27,28]. Importantly, BACE has been identified as a protease responsible for the cleavage and secretion of Golgi-resident ST6Gal I [29]. The mechanisms underlying cleavage are complicated, and have not been well characterized. Soluble forms of glycosyltransferases exist in the plasma of patients with certain diseases, and can sometimes be used as biomarkers for these diseases [30-33].

In the present study, we examined IR-induced cleavage and solubilization of ST6Gal I, which is released into the cell culture media of colon cancer cell lines, and sought to identify the protease involved in cleaving ST6Gal I after exposure to IR. We found that BACE1 could be the secretase responsible for IR-induced cleavage of ST6Gal I, and showed that BACE1 mediated cleavage of ST6Gal I decreased ST6Gal I -mediated cancer cell migration. Additionally, the soluble form of ST6Gal I possesses sialyltransferase enzymatic activity, but unlike the Golgi-associated form, did not sialylate integrin $\beta 1$ to a significant degree, suggesting that soluble ST6Gal I, possibly in cooperation with the Golgibound form, may participate in cancer progression and metastasis before being secreted from cancer cells, independent of intergrin $\beta 1$-sialylation.

\section{Materials and methods}

\section{Cell culture}

SW480 and SW48 human colorectal carcinoma cells were grown in RPMI media supplemented with heatinactivated $10 \%$ fetal bovine serum and antibiotics. CT26 mouse colorectal carcinoma cells were cultured in Dulbecco's modified Eagle's medium (DMEM) supplemented with heat-inactivated $10 \%$ fetal bovine serum and antibiotics.

\section{Plasmids and transfection}

For transient transfection experiments, expression constructs of C-terminally Flag-tagged wild-type (WT; p3 $\times$ Flag-ST6Gal I) ST6Gal I and N-terminally deleted (HAtagged) ST6Gal I (ST6Gal I $\Delta \mathrm{N}$ ) were created as described previously [23]. An N-terminally Flag-tagged double-mutant, ST6Gal I (L37A/K40A) was created by site-directed mutagenesis [34]. Predesigned small interfering RNA (siRNA) for ST6Gal I was purchased from Dharmacon (Lafayette, CO, USA). BACE-1 was targeted with the siRNA duplex 5'-AGA UCC UGU CCA UUG AU CUC CAC CC-3' and 5'-GGG UGG AGA UCA AUG GAC AGG AUC U-3' [35]. A BACE-1 expression plasmid (pcDNA3.1/BACE-1) was generously provided by Dr. Sul-Hee Chung (Inje University, Pusan, Korea) [36]. Cells were transfected with plasmids using LipofectAMINE 2000 (Invitrogen, Carlsbad, CA, USA) as described by the manufacturer.

\section{Irradiation}

Cells were exposed to $\gamma$-irradiation using a ${ }^{137} \mathrm{Cs} \gamma$-ray source (Atomic Energy of Canada, Mississauga, ON, Canada) at a dose rate of $3.81 \mathrm{~Gy} / \mathrm{min}$. 
Immunoblot, lectin affinity assay, and immunoprecipitation

Protein levels were detected using the following commercial antibodies: anti-integrin $\beta 1$ (BD Biosciences, Franklin Lakes, NJ, USA); anti-phospho-p130CAS, anti-phosphoSrc, anti-HA, and anti-Myc (Cell Signaling Technology, Danvers, MA, USA); anti-Flag (Sigma); and anti-ST6Gal I and anti-BACE-1 (IBL, Japan). For detection of sialylated proteins, cell lysates were incubated with biotinylated SNA (Sambucus Nigra lectin; Vector Laboratories, Burlingame, CA, USA), and protein-lectin complexes were precipitated with avidin-coated protein A-agarose (Sigma).

\section{Flow cytometry}

Cells were detached with trypsin/EDTA at the indicated times and stained with fluorescein isothiocyanate (FITC)-conjugated SNA (FITC-SNA) or Maackia amurensis (FITC-MAA) lectin (Vector Laboratories, Burlingame, CA, USA) for detection of $\alpha 2,6$ and $\alpha 2,3$ sialylation, respectively. After staining, fluorescence intensity was analyzed by fluorescence-activated cell sorting (FACS).

Reverse transcription-polymerase chain reaction (RT-PCR) Total RNA was isolated with TRI reagents (Molecular Research Center), and reverse transcription was done using Omniscript transcriptase (Qiagen) under the following thermocycling conditions: 30 cycles of $95^{\circ} \mathrm{C}$ for 5 min (denaturing), $60^{\circ} \mathrm{C}$ for $30 \mathrm{~s}$ (annealing), and $72^{\circ} \mathrm{C}$ for 30 seconds (extension). The primer sequences used were as follows: human BACE1, 5'-GGT GGA GAT CAA TGG ACA GG-3' (sense) and 5'-CGT GGA TGA CTG TGA GAT GG-3' (antisense); mouse Bace1, 5'GCA GAC CCA CAT TCC GAA CA-3' (sense) and 5'GCC ACT GTC CAC GAT GCT CTT-3' (anti-sense); and GAPDH, 5'-CAT GGA GAA GGC TGG GGC TCA TTT-3' (sense) and 5'-CGC CAG TAG AGG CAG GGA TGA TGT-3' (antisense).

\section{In vitro migration assay}

Cell migration assays were performed using a Boyden chamber as previously described [24]. Cells were plated on the upper side of a collagen treated, polycarbonate membrane separating two chambers of 6.5 -mm Transwell culture plates (Costar, Corning, NY, USA). After 24 hours, cells on the upper face of the membrane were scraped using a cotton swab and cells that had migrated to the lower face of the membrane were stained with DiffQuick (Baxter Scientific, Deerfield, IL, USA) WrightGiemsa Solution.

\section{Soluble ST6Gal I ELISA}

Cell culture media were concentrated using Centricon centrifugal filter devices (Millipore, Billerica, MA, USA) and then analyzed for soluble ST6Gal I levels using an $\alpha 2,6$ sialyltransferase sandwich enzyme-linked immunosorbent assay (ELISA) kit (IBL, Japan), according to the manufacturer's protocol.

\section{Statistical analysis}

Data are expressed as means \pm standard deviations (SDs). Statistical significance was determined using Student's $t$-test for comparisons between two means. The null hypothesis was rejected in cases where $p$-values were $<0.05$.

\section{Results}

Inhibition of ST6Gal I cleavage by knockdown of BACE1 increases sialylation of cell surface protein

In our previous study, we found that IR exposure induces ST6Gal I cleavage and produces a soluble form of ST6Gal I in SW480 colon cancer cells [23]. To support the result of our prior work, we next examined the expression of soluble ST6 Gal I in SW48 cells (ST6 Gal $\left.\mathrm{I}^{-/-}\right)$by transiently transfecting these cells with expression vectors for Golgi-anchored (N-terminally Flagtagged and C-terminally Myc-tagged) of ST6Gal I and exposing them to IR. As shown in Figure 1A, both the Golgi-anchored and soluble forms of ST6Gal I were expressed in SW48 colon cancer cells (Figure 1A). Because it had previously been demonstrated that sialyltransferases are cleaved by cathepsin-like protease or BACE $[3,29,34,37]$, we sought to identify the target protease(s) responsible for cleavage of ST6Gal I using various protease inhibitors in SW480 vector-control and ST6Gal I-overexpressing cells. Inhibitors of $\beta$-secretase and $\gamma$-secretase increased the protein levels of the uncleaved, proform of ST6Gal I, whereas cathepsin B and L (CTS) inhibitors did not (Figure 1B). In addition, $\alpha 2,6$ sialylation level at the cell surface, detected by FITC-conjugated SNA, was also increased by treatment with $\beta$-secretase or $\gamma$-secretase, but not CTS, inhibitors. In contrast, $\alpha 2,3$ sialylation at the cell surface, detected by FITC-MAA, was not altered by these inhibitors (Figure $1 C)$, suggesting that $\beta$-secretase or $\gamma$-secretase might be responsible for the cleavage of ST6Gal I in SW480 colon cancer cells. Next, because BACE1 is reported to be the protease responsible for the cleavage of ST6Gal 1 $[34,35]$, we tested the effects of siRNA-mediated BACE1 knockdown. We observed a concomitant increase in the level of non-cleaved ST6Gal I protein and sialylation of integrin $\beta 1$, a substrate of ST6Gal I (Figure 1D). Moreover, knockdown of BACE1 expression augmented the sialylation on the surface of these cells. Interestingly, BACE1 overexpression did not specifically inhibit cell surface sialylation (and may have slightly increased it) compared with control levels (Figure 1E), suggesting that BACE1-mediated cleavage of ST6Gal I only slightly 

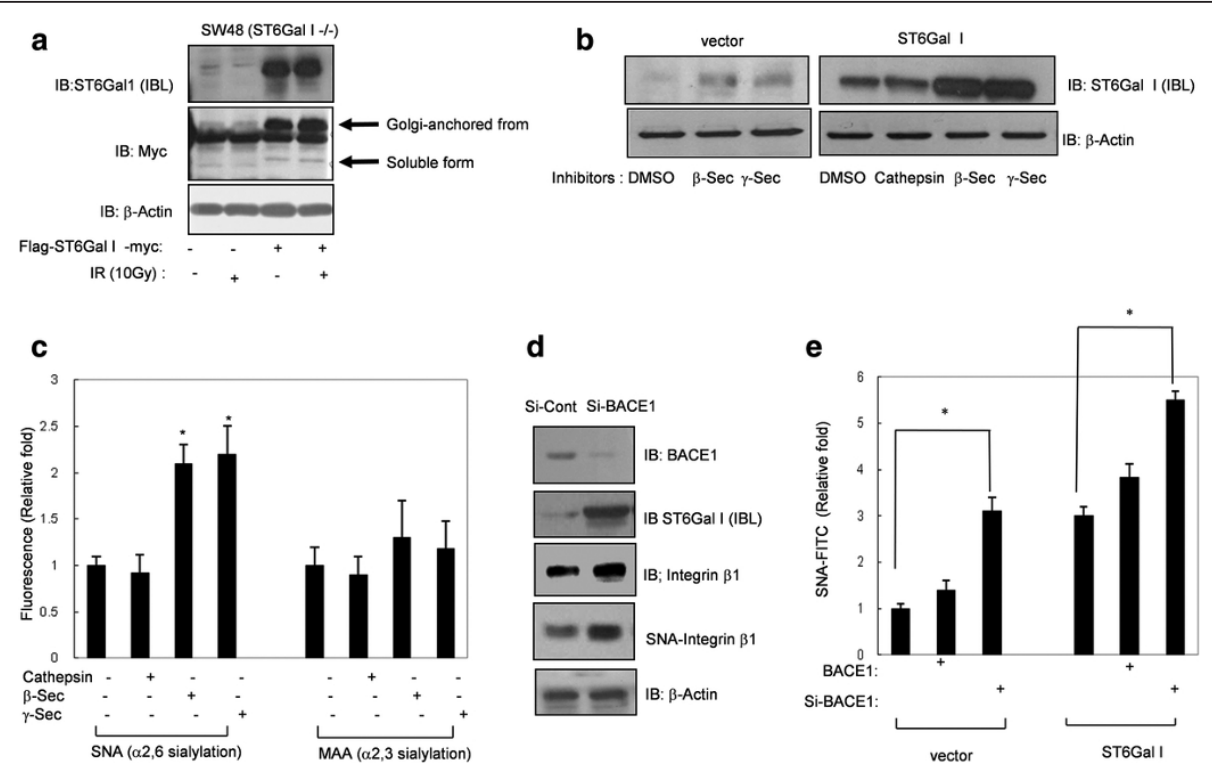

Figure 1 Inhibition of BACE1 stabilizes ST6Gal I and increases cell surface sialylation. A. SW48 (ST6Gal I-negative) colon cancer cells were transiently transfected with an N-terminally Flag-tagged ST6Gal I expression plasmid, and 24 hours later, cells were exposed to IR (10 Gy). Cell lysates were assessed for expression of ST6Gal I by immunoblotting with an ST6Gal I antibody. B. SW480 vector-control cells and cells stably expressing ST6Gal I were treated with inhibitors of $\beta$-secretase, $\gamma$-secretase, or cathepsin B\&L at a concentration of $20 \mu \mathrm{M}$. After incubating for 24 hours, cells were harvested and ST6Gal I levels were analyzed by immunoblotting with an anti-ST6Gal I antibody. C. Cell surface $\alpha 2,6$ sialylation (SNA-FITC) and $\alpha 2,3$ sialylation (MAA-FITC) were analyzed by FACS after 24 hours of treatment with $20 \mu \mathrm{M} \beta$-secretase or $\gamma$-secretase inhibitors. D. SW480 cells were transfected with siRNA against BACE1, and the levels of BACE1, ST6Gal I, and integrin $\beta 1$ protein were assessed by immunoblotting. Sialylation of integrin $\beta 1$ was assayed by SNA lectin affinity assay. E. SW480 vector-control cells and cells stably expressing ST6Gal I were treated with Si-BACE1 or transfected with a BACE1 expression plasmid. Then, $\alpha 2,6$ sialylation (SNA-FITC) of the cell surface was analyzed by FACS. Data are presented as means \pm SDs of three replicates $\left({ }^{*} p<0.05\right.$ vs. the corresponding control).

shows the sialylation potential of ST6Gal I. Taken together, these results suggest that BACE1 may be responsible for cleavage of ST6Gal I.

The non-cleavable, double-mutant ST6Gal I (L37A/K40A) increases $\alpha 2,6$ sialylation at the cell surface

It was previously demonstrated that replacement of ST6Gal I residues Leu37 and Lys40 with alanine significantly decreases BACE1-mediated cleavage of ST6Gal I [34]. Therefore, we prepared a N-terminally Flag-tagged and C-terminally myc-tagged, non-cleavable ST6Gal I (L37A/K40A) mutant and an HA-tagged, N-terminally truncated soluble form of ST6Gal I $(\Delta \mathrm{N}$, amino acids 43-403) lacking the protease-specific recognition motif (Figure 2A). We then transiently transfected cells with ST6Gal I WT, L37A/K40A or $\Delta \mathrm{N}$ constructs, and examined ST6Gal I protein expression by immunoblotting (Figure 2A) and measured ST6Gal I enzymatic activity and sialylation of cell surface proteins 24 hours later. We found that the enzymatic activity of both the noncleavable L37A/K40A mutant and the $\Delta \mathrm{N}$ soluble form was similar to that of WT (Figure 2B). In addition, L37A/K40A increased cell surface sialylation to a similar extent as WT. However, in the case of $\Delta \mathrm{N}$, which also had enzymatic catalytic activity, cell surface glycoproteins were barely sialylated compared to WT or L37A/K40A (Figure 2C). These results show that the non-cleavable form of ST6Gal I has enzymatic activity and increases $\alpha 2,6$ sialylation at the cell surface to the same extent as WT. However, it was suggested that the sialylation targets of the soluble form of ST6Gal I were not cell surface proteins. This result may be due to the fact that there isn't much CMP-sialic acid substrate on the outside of the cell.

\section{Non-cleaved form ST6Gal I shows integrin $\beta 1$ dependent cell migration}

Integrin $\beta 1$, a known substrate of ST6Gal I $[4,38]$, together with associated adaptor molecules, such as p130CAS, plays a role in promoting the migration of cancer cells [39-41]. We previously reported that integrin $\beta 1$ sialylation and its associated cancer cell migration-related signaling activity are increased by IRinduced ST6Gal I [22,23,26]. The phenomena described above prompted us to compare the action of WT and non-cleavable L37A/K40A on integrin $\beta 1$ sialylation, integrin $\beta 1$-mediated signaling, and cell migration. As shown in Figure 3A, transfection with L37A/K40A increased the Tyr410-phosphorylated (active) form of p130CAS (p-p130CAS ${ }^{\mathrm{Y} 410}$ ) and sialylation of integrin $\beta 1$ 

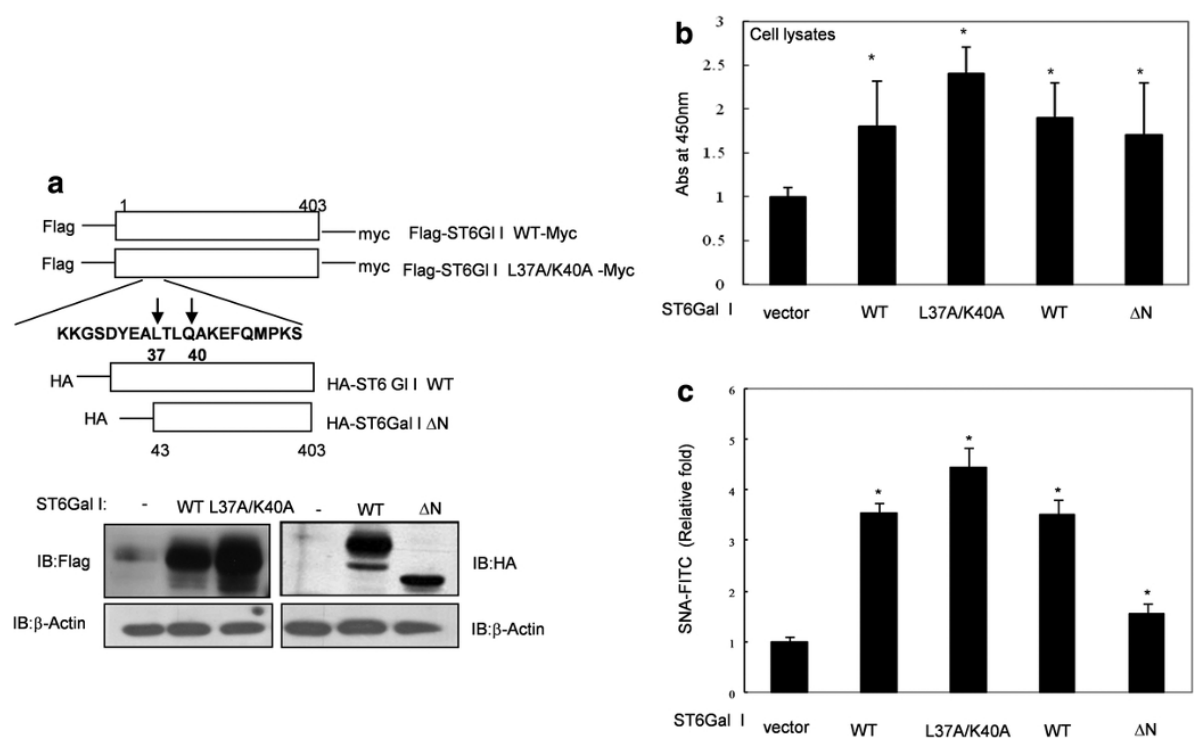

Figure 2 Construction of ST6Gal I expression plasmids and their effect on $\alpha 2,6$ sialylation at the cell surface. A. Expression plasmids for full-length WT ST6Gal I (WT), double-mutant ST6Gal I (L37A/K40A) and N-terminal ST6Gal I deletion mutant ( $\triangle$ N, containing amino acids 43-403) were constructed. WT and L37A/K40A were N-terminally tagged with Flag; $\triangle N$ was tagged with HA. Expression of each construct was confirmed by immunoblotting. SW480 cells were transfected with WT, L37A/K40A, or $\triangle N$. Then, ST6Gal I enzymatic activity was measured by ELISA (B), and cell surface sialylation was detected by FACS $(\mathbf{C})$. Data are presented as means \pm SDs of three replicates $\left({ }^{*} p<0.05\right.$ vs. the corresponding control).

to a greater degree than did the WT form. Next, we further examined the effects of BACE1 on ST6Gal I cleavage by co-transfecting SW480 cells with BACE1 and WT or L37A/K40A forms of ST6Gal I. These experiments showed that the Golgi-anchored WT proform was decreased to a greater extent by co-transfection of BACE1 compared with the non-cleavable L37A/ K40A form (Figure 3B). We then extended our investigation of the roles of BACE1 in ST6Gal I-mediated migration of cancer cells by co-transfecting SW480 and CT26 cells with BACE1 and WT or L37A/K40A. Cell migration was increased to a much lesser extent by coexpression of BACE1 and WT compared with coexpression of BACE1 and L37A/K40A (Figure 3C). These findings demonstrate that BACE- 1 is capable of cleaving the Golgi-membrane anchored ST6Gal I, and indicate that BACE-1-induced cleavage of ST6Gal I results in decreased cell migration.

The soluble form of ST6Gal I is partially involved in the migration of colon cancer cells

Next, we compared the effects of ST6Gal I WT and $\Delta N$ on integrin $\beta 1$ sialylation, integrin $\beta 1$-mediated signaling, and cell migration. Expression of either WT or $\Delta \mathrm{N}$ increased the levels of the Tyr410-phosphorylated (active) form of p130CAS (p-p130CAS ${ }^{\mathrm{Y} 410}$; Figure 4A) and enhanced cell migration (Figure 4B). Although the $\Delta \mathrm{N}$ soluble form had no significant effects on sialylation of the cell surface glycoprotein integrin $\beta 1$, it significantly induced p130CAS phosphorylation and migration of SW480 cells (Figure 4A and 4B) and CT26 cells (data not shown), similar to the results of our previous study $[23,26]$. These findings suggest that the soluble form of ST6Gal I could be involved in p130CAS signaling and migration of colon cancer cells, which, in turn, may be independent of integrin $\beta 1$ sialylation.

\section{BACE-1 mediated cleavage and secretion of ST6Gal I are increased by IR}

Our previous study indicated that IR induces ST6Gal I cleavage and secretion of a soluble form of ST6Gal I [23]. Because it has been suggested that BACE1 is a protease of ST6Gal I, we tested the effect of IR on BACE1 expression. Importantly, IR (10 Gy) increased the expression of BACE1 at both mRNA and protein levels in SW480 and CT26 colon cancer cell lines (Figure 5A). Next, we examined whether IR induced ST6Gal I cleavage and produced a soluble form of ST6Gal I. As shown in Figure 5B, IR increased cleavage of ST6Gal I and knockdown of BACE1 inhibited this cleavage. In both SW480 and CT26 cell lines, the enzymatic activity of ST6Gal I in culture media was increased compared to that in control cells after exposure of vector control or ST6Gal I-overexpressing cells to IR (Figure 5C). However, even though IR alone slightly increased cell surface protein sialylation which might be induction of ST6Gal 


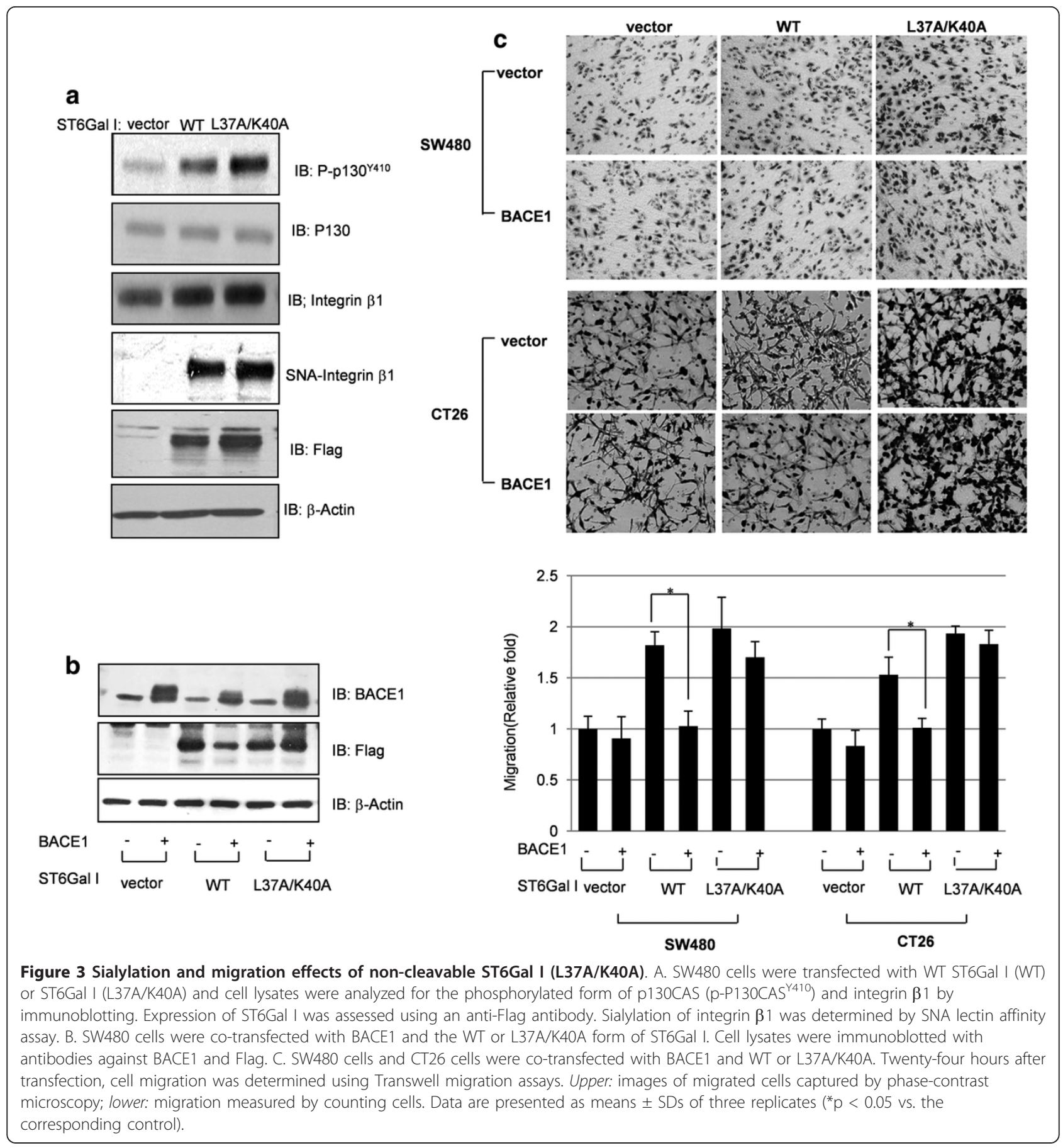

I by IR [23,24], Golg-anchored ST6Gal I-mediated increase of cell surface sialylation was not augmented by IR (Figure 5D). We also checked the effect of IR on ST6Gal I-induced cell migration. ST6Gal I enhanced CT26 cell migration, an effect that was partially inhibited by IR. These results indicate that IR acted through induction of BACE1 expression to induce cleavage of ST6Gal I protein to a soluble form that retained sialyltransferase activity. ST6Gal I-induced cell surface sialylation and migration were partially inhibited by IR, an effect that might be due to cleavage of ST6Gal I (Figure $5 \mathrm{E})$.

\section{Discussion}

In the present study, we showed that IR induced cleavage and secretion of the soluble form of ST6Gal I by increasing BACE-1 expression, an effect that may contribute to IR-induced migration of colon cancer cells, 
a

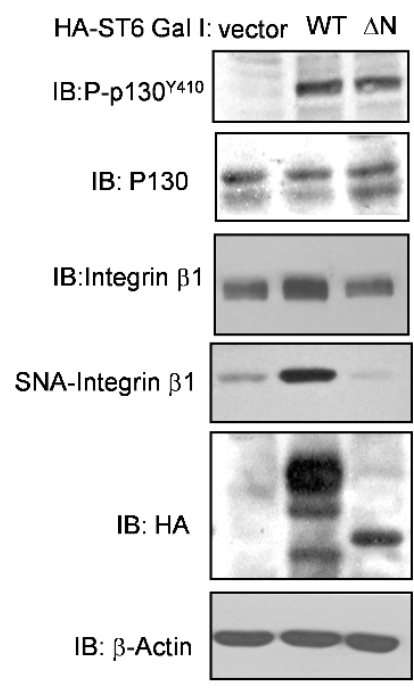

b

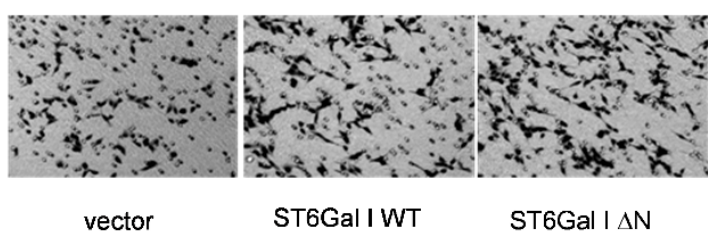

vector
ST6Gal I $\Delta \mathrm{N}$

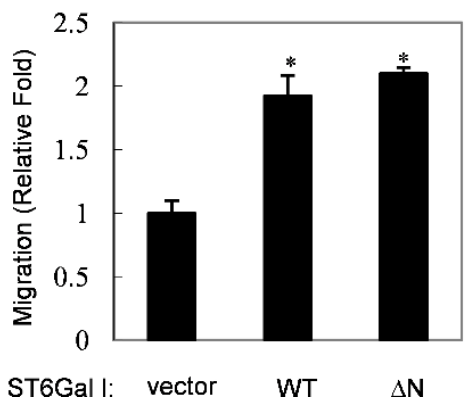

Figure 4 The soluble form of ST6Gal I increases the migration of colon cancer cells. A. SW480 cells were transfected with WT ST6Gal I $(\mathrm{WT})$ or ST6Gal I- $\Delta N(\Delta N)$, and cell lysates were analyzed for the phosphorylated form of p130CAS (p-P130CAS ${ }^{Y 410}$ ) and integrin $\beta 1$ by immunoblotting. Sialylation of integrin $\beta 1$ was determined by SNA lectin affinity assay. B. After transfection with the WT or $\triangle \mathrm{N}$ form of ST6Gal I, cell migration was determined using Transwell migration assays. Upper: images of migrated cells captured by phase-contrast microscopy; lower: migration measured by counting cells. Data are presented as means \pm SDs of three replicates $\left({ }^{*} \mathrm{p}<0.05 \mathrm{vs}\right.$. the corresponding control).

which phenomena is independent of integrin $\beta 1$ sialylation that was usually mediated by Golgi-anchored ST6Gal I.

Our previous study suggested that IR increases the expression of ST6Gal I, which, in turn, is involved in radioresistance and radiation-induced migration via sialylation of integrin $\beta 1[22,23,26]$. Another interesting issue raised previously is that IR increases ST6Gal I cleavage and secretion into culture media [23]. While the current data indicate that the Golgi is the main site of sialylation by ST6Gal I in most cells, the fact that soluble forms of ST6Gal I are detected in body fluids and media from cultured cells has raised questions concerning the functions of this extracellular ST6Gal I. It is known that ST6Gal I is not the only transferase that exists in a soluble form; other glycosyltransferases are also detected in the systemic circulation of cancer patients, where they are associated with disease severity and poor prognosis. It has also been suggested that soluble ST6Gal I may be a potential biomarker for the clinical evaluation of colorectal cancer, highlighting the importance of elucidating the function of soluble ST6Gal I, particularly in the radiation-induced migration of cancer cells.

BACE-1, which is a crucial protease in the pathogenesis of Alzheimer's disease, is highly expressed in the brain, but it is also expressed at low levels in peripheral tissues. Information regarding BACE-1 expression and functions outside of the brain is limited. Furthermore, the physiological importance of BACE-1 in cancer progression, metastasis, and responsiveness to radiation remains largely unknown. Evidence that BACE-1 is one of the proteases responsible for the cleavage of ST6Gal I was provided by experiments employing a $\beta$-secretase inhibitor or siRNA-mediated knockdown of BACE-1 (Figure 1). The results of these experiments suggest that BACE1 activity involved in cleavage of ST6Gal I from the Golgi membrane and inducing secretion.

Interestingly, inhibition of $\gamma$-secretase also increased ST6Gal I levels and cell surface sialylation. Although BACE-1 expression levels could be a major determinant of ST6Gal I cleavage, other regulatory mechanisms might also affect cleavage, secretion, or sorting of ST6Gal I to critical subcellular localizations where ST6Gal I could encounter proteases. Such BACE-1independent mechanisms might also regulate ST6Gal I function.

In colon cancer cells, IR increased the expression of BACE1, which is involved in IR-mediated cleavage of ST6Gal I (Figure 5). To address the detailed mechanisms linking ST6Gal I to cancer cell migration, we established non-cleavable (L37A/K40A) and soluble $(\Delta \mathrm{N})$ variants of ST6Gal I that retained catalytic activity (Figure 2). Cleaved ST6Gal I was secreted into the 

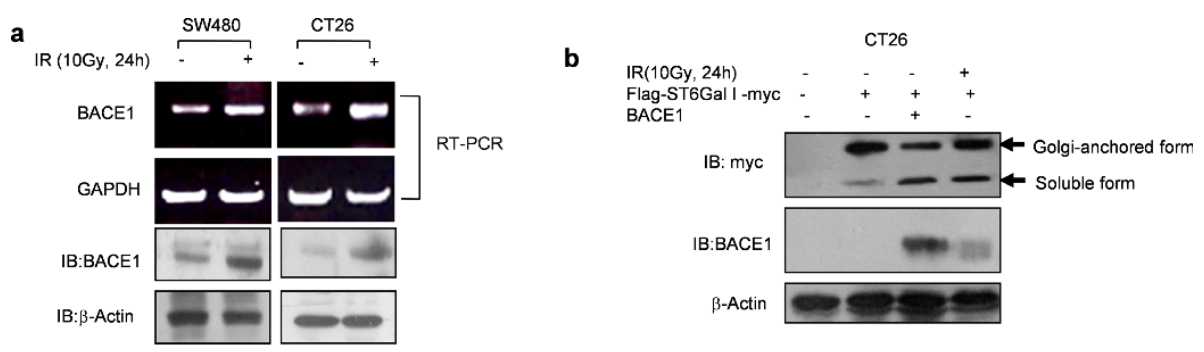

c

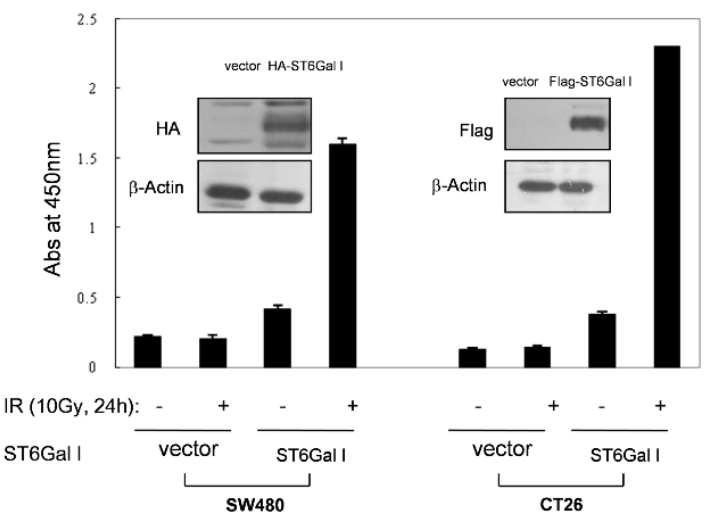

d

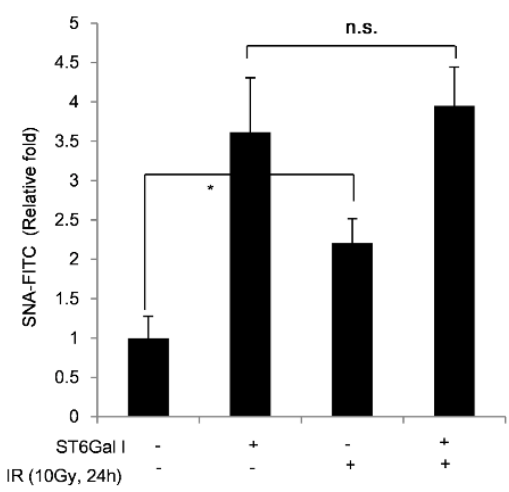

$\mathbf{e}$

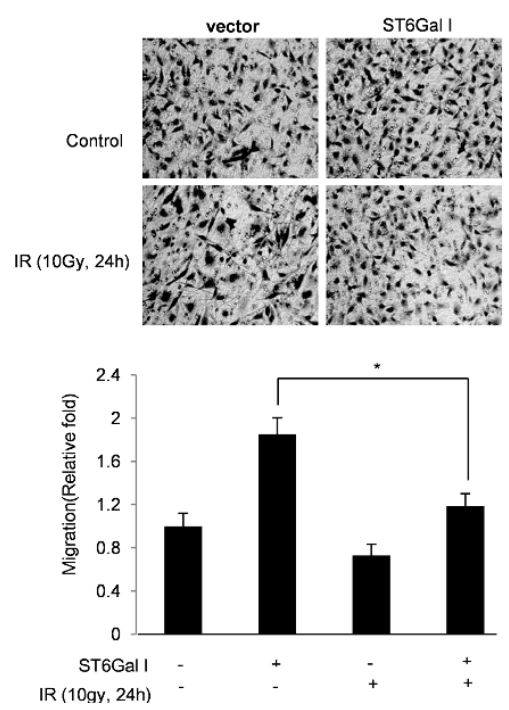

Figure 5 IR-induced BACE-1 mediates cleavage of ST6Gal I, and inhibits sialylation of cell surface molecules and colon cancer cell migration. A. Following irradiation of SW480 and CT26 cells, BACE1 expression levels were determined by RT-PCR and immunoblotting. CT26 vector control cells and cells stably expressing ST6Gal I were exposed to 10 Gy of IR. B. The soluble form of ST6Gal I was analyzed after cotransfection with WT ST6Gal I and BACE1, or after exposure of IR (10 Gy, 48 h) to WT ST6Gal I -transfected cells. C. SW480 and CT26 colorectal carcinoma cell lines were stably transfected with ST6Gal I and exposed to 10 Gy of IR. After 24 hours, culture media were harvested and ST6Gal I activity was assayed by ELISA. Data are presented as means \pm SDs from three replicates. CT26 vector control cells and cells stably expressing ST6Gal I were exposed to $10 \mathrm{~Gy}$ of IR. After $24, \alpha 2,6$ sialylation of cell surface was analyzed by flow cytometry. Data are presented as means \pm SDs of three replicates. ${ }^{*} p<0.05$. n.s., not significant. (D). Cell migration was determined using Transwell migration assays (E). Upper: images of migrated cells captured by phase-contrast microscopy; lower: migration measured by counting cells. Data are presented as means \pm SDs of three replicates $\left({ }^{*} \mathrm{p}<0.05\right.$ vs. the corresponding control). 
culture media after exposure to radiation (Figure 5), and the truncated soluble $\Delta \mathrm{N}$ form retained sialyltransferase activity (Figure 2). We had predicted that the soluble form of ST6Gal I was merely a byproduct of the Golgianchored proform of ST6Gal I that was excreted into the extracellular milieu after exposure of cells to radiation. However, ST6Gal I- $\Delta \mathrm{N}$ also induced cellular migration (Figure 4), suggesting that the soluble form of ST6Gal I could function in tumor cell migration with unknown mechanism. In addition, it was proposed that soluble ST6Gal I would catalyze the sialylation of soluble glycoproteins in the trans-Golgi network, or secretory vesicles [35]. These phenomena would be related with sialylation of unknown glycoproteins in intracellular region and subsequently have effect on the process of cell migration. An examination of integrin $\beta 1$, which is involved in IR-mediated sialylation and tumor migration $[23,26]$, showed that the soluble form of ST6Gal I did not affect the sialylation of integrin $\beta 1$ (Figure 4). However, cleavage of ST6Gal I by IR inhibited IRinduced migration (Figure 5), indicating that increase of integrin $\beta 1$-mediated migration by Golgi-anchored ST6Gal I is more potent phenomena than that of soluble form ST6Gal I.

Therefore, it could be suggested that a transfer of sialic acids that is independent of integrin $\beta 1$ sialylation might be involved in soluble ST6Gal I-mediated migration and activation of p130CAS.

\section{Conclusion}

Collectively, our data suggest that glycoconjugation by the Golgi form of ST6Gal I, possibly in cooperation with soluble ST6Gal I, may be involved in the process of cancer metastasis, especially after radiation therapy.

\section{Funding}

This work was supported by the Nuclear Research and Development Program through a National Research Foundation of Korea (NRF) grant funded by the Korean government (Ministry of Education, Science and Technology; grant code: M2AMA006), and by a grant from the Korea Healthcare Technology R\&D Project, Ministry for Health, Welfare \& Family Affairs (grant code: A100627). This work was also supported by the Ewha Global Top5 Grant 2011 of Ewha Womans University.

\footnotetext{
Author details

${ }^{1}$ Division of Radiation Effects, Korea Institute of Radiological and Medical Sciences, Seoul 139-706, South Korea. ${ }^{2}$ College of Life Sciences and Biotechnology, Korea University, 1, 5-ka, Anamdong, Sungbuk-gu, Seoul 136701, South Korea. ${ }^{3}$ College of Pharmacy \& Division of Life \& Pharmaceutical Sciences, Ewha Womans University, 11-1 Daehyun-Dong, Seodaemun-Gu, Seoul 120-750, South Korea.
}

\section{Authors' contributions}

ML and JJP performed experiments. YGK and YSL designed, analyzed data, and wrote paper. All authors have read and approved the final manuscript.

\section{Competing interests}

The authors declare that they have no competing interests.

Received: 1 December 2011 Accepted: 27 March 2012

Published: 27 March 2012

\section{References}

1. Dall'Olio F: The sialyl-alpha2,6-lactosaminyl-structure: biosynthesis and functional role. Glycoconj J 2000, 17:669-676.

2. Dall'Olio F, Chiricolo M, Ceccarelli C, Minni F, Marrano D, Santini D: Betagalactoside alpha2,6 sialyltransferase in human colon cancer: contribution of multiple transcripts to regulation of enzyme activity and reactivity with Sambucus nigra agglutinin. Int J Cancer 2000, 88:58-65.

3. Lammers $G$, Jamieson JC: The role of a cathepsin D-like activity in the release of Gal beta 1-4GIcNAc alpha 2-6-sialyltransferase from rat liver Golgi membranes during the acute-phase response. The Biochemical journal 1988, 256:623-631.

4. Seales EC, Jurado GA, Brunson BA, Wakefield JK, Frost AR, Bellis SL: Hypersialylation of beta1 integrins, observed in colon adenocarcinoma, may contribute to cancer progression by up-regulating cell motility. Cancer Res 2005, 65:4645-4652.

5. Le Marer N, Stehelin D: High alpha-2,6-sialylation of N-acetyllactosamine sequences in ras-transformed rat fibroblasts correlates with high invasive potential. Glycobiology 1995, 5:219-226.

6. Lise M, Belluco C, Perera SP, Patel R, Thomas P, Ganguly A: Clinical correlations of alpha2,6-sialyltransferase expression in colorectal cancer patients. Hybridoma 2000, 19:281-286.

7. Poon TC, Chiu CH, Lai PB, Mok TS, Zee B, Chan AT, Sung JJ, Johnson PJ: Correlation and prognostic significance of beta-galactoside alpha-2,6sialyltransferase and serum monosialylated alpha-fetoprotein in hepatocellular carcinoma. World J Gastroenterol 2005, 11:6701-6706.

8. Wang PH, Lee WL, Juang CM, Yang YH, Lo WH, Lai CR, Hsieh SL, Yuan CC: Altered mRNA expressions of sialyltransferases in ovarian cancers. Gynecologic oncology 2005, 99:631-639.

9. Stewart JF, Rubens RD, Hoare S, Bulbrook RD, Kessel D: Serum sialyl transferase levels in patients with metastatic breast cancer treated by chemotherapy. British journal of cancer 1982, 46:208-212.

10. Bernacki RJ, Kim U: Concomitant elevations in serum sialytransferase activity and sialic acid content in rats with metastasizing mammary tumors. Science (New York, N.Y 1977, 195:577-580.

11. Gessner P, Riedl S, Quentmaier A, Kemmner W: Enhanced activity of CMPneuAc: Gal beta 1-4GIcNAc:alpha 2,6-sialyltransferase in metastasizing human colorectal tumor tissue and serum of tumor patients. Cancer letters 1993, 75:143-149.

12. Ganzinger $U$, Moser $K$ : Sialyl transferase activity: a serum enzyme marker in the follow-up of cancer patients. Recent results in cancer research. Fortschritte der Krebsforschung 1979, 67:50-55.

13. Herrmann WP, Gielen W: Sialyltransferase levels and sialic acid concentrations in sera of patients with malignant melanomas. Archives of dermatological research 1979, 265:321-329.

14. Kawaguchi T: Cancer metastasis: characterization and identification of the behavior of metastatic tumor cells and the cell adhesion molecules, including carbohydrates. Curr Drug Targets Cardiovasc Haematol Disord 2005, 5:39-64.

15. Hakomori S: Tumor malignancy defined by aberrant glycosylation and sphingo(glyco)lipid metabolism. Cancer research 1996, 56:5309-5318.

16. Zhao YY, Takahashi M, Gu JG, Miyoshi E, Matsumoto A, Kitazume S, Taniguchi N: Functional roles of N-glycans in cell signaling and cell adhesion in cancer. Cancer science 2008, 99:1304-1310.

17. Gorelik E, Galili U, Raz A: On the role of cell surface carbohydrates and their binding proteins (lectins) in tumor metastasis. Cancer Metastasis Rev 2001, 20:245-277.

18. Semel AC, Seales EC, Singhal A, Eklund EA, Colley KJ, Bellis SL: Hyposialylation of integrins stimulates the activity of myeloid fibronectin receptors. J Biol Chem 2002, 277:32830-32836. 
19. Seales EC, Jurado GA, Singhal A, Bellis SL: Ras oncogene directs expression of a differentially sialylated, functionally altered beta1 integrin. Oncogene 2003, 22:7137-7145.

20. van der Flier A, Sonnenberg A: Function and interactions of integrins. Cell Tissue Res 2001, 305:285-298.

21. Van Slambrouck S, Grijelmo C, De Wever O, Bruyneel E, Emami S, Gespach C, Steelant WF: Activation of the FAK-src molecular scaffolds and p130Cas-JNK signaling cascades by alpha1-integrins during colon cancer cell invasion. Int J Oncol 2007, 31:1501-1508.

22. Lee M, Park JJ, Lee YS: Adhesion of ST6Gal I-mediated human colon cancer cells to fibronectin contributes to cell survival by integrin beta1mediated paxillin and AKT activation. Oncol Rep 2010, 23:757-761.

23. Lee M, Lee HJ, Bae S, Lee YS: Protein sialylation by sialyltransferase involves radiation resistance. Mol Cancer Res 2008, 6:1316-1325.

24. Lee M, Lee HJ, Seo WD, Park KH, Lee YS: Sialylation of integrin beta1 is involved in radiation-induced adhesion and migration in human colon cancer cells. Int J Radiat Oncol Biol Phys 2010, 76:1528-1536.

25. Jang ER, Ryu M, Park JE, Kim JH, Lee JS, Song K: A new isoquinolinium derivative, Cadein 1, preferentially induces apoptosis in p53-defective cancer cells with functional mismatch repair via a p38-dependent pathway. J Biol Chem 2010, 285:2986-2995.

26. Lee M, Lee HJ, Seo WD, Park KH, Lee YS: Sialylation of integrin beta1 is involved in radiation-induced adhesion and migration in human colon cancer cells. Int J Radiat Oncol Biol Phys 2010, 76:1528-1536.

27. Vassar R, Kovacs DM, Yan R, Wong PC: The beta-secretase enzyme BACE in health and Alzheimer's disease: regulation, cell biology, function, and therapeutic potential. J Neurosci 2009, 29:12787-12794.

28. Vassar R, Bennett BD, Babu-Khan S, Kahn S, Mendiaz EA, Denis P, Teplow DB, Ross S, Amarante P, Loeloff R, et al: Beta-secretase cleavage of Alzheimer's amyloid precursor protein by the transmembrane aspartic protease BACE. Science (New York, N.Y 1999, 286:735-741.

29. Kitazume S, Oka R, Ogawa K, Futakawa S, Hagiwara Y, Takikawa H, Kato M, Kasahara A, Miyoshi E, Taniguchi N, Hashimoto Y: Molecular insights into beta-galactoside alpha2,6-sialyltransferase secretion in vivo. Glycobiology 2009, 19:479-487.

30. Strous GJ: Golgi and secreted galactosyltransferase. $C R C$ critical reviews in biochemistry 1986, 21:119-151.

31. Paone JF, Waalkes TP, Baker RR, Shaper JH: Serum UDP-galactosyl transferase as a potential biomarker for breast carcinoma. Journal of surgical oncology 1980, 15:59-66.

32. De AK, Hardy RE: Elucidation of sialyltransferase as a tumour marker. Indian journal of biochemistry \& biophysics 1990, 27:452-455

33. Futakawa S, Kitazume S, Oka R, Ogawa K, Hagiwara Y, Kinoshita A, Miyashita K, Hashimoto Y: Development of sandwich enzyme-linked immunosorbent assay systems for plasma beta-galactoside alpha2,6sialyltransferase, a possible hepatic disease biomarker. Analytica chimica acta 2009, 631:116-120.

34. Kitazume S, Suzuki M, Saido TC, Hashimoto Y: Involvement of proteases in glycosyltransferase secretion: Alzheimer's beta-secretase-dependent cleavage and a following processing by an aminopeptidase. Glycoconjugate journal 2004, 21:25-29.

35. Sugimoto I, Futakawa S, Oka R, Ogawa K, Marth JD, Miyoshi E, Taniguchi N, Hashimoto Y, Kitazume S: Beta-galactoside alpha2,6-sialyltransferase I cleavage by BACE1 enhances the sialylation of soluble glycoproteins. A novel regulatory mechanism for alpha2,6-sialylation. The Journal of biological chemistry 2007, 282:34896-34903.

36. Lee HW, Seo HS, Ha I, Chung SH: Overexpression of BACE1 stimulates spontaneous basal secretion in PC12 cells. Neuroscience letters 2007, 421:178-183.

37. Pietras RJ, Szego CM, Mangan CE, Seeler BJ, Burtnett MM, Orevi M: Elevated serum cathepsin $B 1$ and vaginal pathology after prenatal DES exposure. Obstetrics and gynecology 1978, 52:321-327.

38. Seales EC, Shaikh FM, Woodard-Grice AV, Aggarwal P, McBrayer AC, Hennessy KM, Bellis SL: A protein kinase C/Ras/ERK signaling pathway activates myeloid fibronectin receptors by altering beta1 integrin sialylation. J Biol Chem 2005, 280:37610-37615.

39. Cordes N, Blaese MA, Meineke V, Van Beuningen D: Ionizing radiation induces up-regulation of functional beta1-integrin in human lung tumour cell lines in vitro. Int J Radiat Biol 2002, 78:347-357.

40. Cordes N, Park CC: beta1 integrin as a molecular therapeutic target. Int J Radiat Biol 2007, 83:753-760.
41. Cordes N, Seidler J, Durzok R, Geinitz H, Brakebusch C: beta1-integrinmediated signaling essentially contributes to cell survival after radiationinduced genotoxic injury. Oncogene 2006, 25:1378-1390.

doi:10.1186/1748-717X-7-47

Cite this article as: Lee et al:: Cleavage of ST6Gal I by Radiation-Induced BACE1 Inhibits Golgi-Anchored ST6Gal I-Mediated Sialylation of Integrin $\beta 1$ and Migration in Colon Cancer Cells. Radiation Oncology 2012 7:47.

\section{Submit your next manuscript to BioMed Central and take full advantage of:}

- Convenient online submission

- Thorough peer review

- No space constraints or color figure charges

- Immediate publication on acceptance

- Inclusion in PubMed, CAS, Scopus and Google Scholar

- Research which is freely available for redistribution

Submit your manuscript at www.biomedcentral.com/submit
Biomed Central 